\title{
Capital social et concurrence de statut
}

Le champ de la restauration commerciale

\section{Fabien Éloire}

\section{OpenEdition}

\section{Journals}

Édition électronique

URL : http://journals.openedition.org/ress/959

DOI : $10.4000 /$ ress.959

ISSN : 1663-4446

Éditeur

Librairie Droz

\section{Édition imprimée}

Date de publication : 15 décembre 2011

Pagination : 7-41

ISBN : 978-2-600-01575-2

ISSN : 0048-8046

\section{Référence électronique}

Fabien Éloire, «Capital social et concurrence de statut », Revue européenne des sciences sociales [En ligne], 49-2 | 2011, mis en ligne le 01 janvier 2015, consulté le 19 avril 2019. URL : http:// journals.openedition.org/ress/959; DOI : 10.4000/ress.959 


\title{
CAPITAL SOCIAL \\ ET CONCURRENCE DE STATUT \\ LE CHAMP DE LA RESTAURATION COMMERCIALE
}

FABIEN ÉLOIRE

Université Lille-I - Clersé

fabien.eloire@univ-lillel.fr

\begin{abstract}
Résumé. De nombreux de travaux de sociologie économique mettent en lumière l'importance du capital social dans le monde des affaires. Ces travaux insistent surtout sur la dimension instrumentale du capital social: dans quelle mesure est-il directement profitable? Dans cet article, nous proposons un autre cadre d'analyse. Nous insistons sur l'idée originelle de Pierre Bourdieu selon laquelle le capital social est un «méta-capital», dont les effets sont principalement indirects, susceptibles de multiplier les effets des autres formes de capital (à savoir économique, symbolique et culturel). Nous illustrons cette idée à partir d'une enquête de terrain auprès d'environ trois cents propriétaires de restaurants installés sur une même aire géographique (l'agglomération lilloise, dans le Nord de la France). Nous mobilisons l'analyse en termes de champ, que nous couplons à une analyse de réseaux sociaux. Nous montrons qu'il existe une interdépendance entre capital social, réussite économique et la position objective des restaurateurs dans la structure du champ.
\end{abstract}

Mots-clés: capital social, concurrence, réseaux sociaux, champ, restauration, gastronomie

\begin{abstract}
Many studies in economic sociology highlight the importance of social capital for business. This literature usually insists on the instrumental dimension of social capital: how is social capital directly profitable? In this article, I aim at proposing another framework of analysis. I emphasize on Pierre Bourdieu's idea of social capital as a "meta-capital", which effects are mainly indirect effects, i.e. able to multiply the effects of the other forms of capital. I illustrate this idea thanks to the data of an empirical survey on about three hundred restaurants' owners located in the same area (Lille, in the North of France). I use both field and social network analysis. I underline the fact that it exists some interdependence between social capital, economic profit, and the restaurants owners' objective position inside the field structure.
\end{abstract}

Keywords: social capital, competition, social networks, field analysis, catering, gastronomy 
Après trente années d'un développement remarquable et transdisciplinaire au sein des sciences sociales (sociologie, sciences politiques, économie), le «capital social» semble être devenu, tout comme l'«encastrement», un concept «valise» à propos duquel on retient surtout la concurrence entre les définitions, plutôt que les définitions elles-mêmes. Lorsqu'on s'intéresse à l'histoire de ce concept, on rencontre tous les grands problèmes théoriques transversaux aux disciplines des sciences sociales : la relation entre micro et macro, entre approches individualiste et holiste, entre choix rationnels et structures sociales (Ponthieux, 2006). En sociologie, le succès du capital social s'est appuyé d’une part sur le développement de l’analyse de réseaux sociaux, d’autre part sur le renouveau de la sociologie économique. Mais c'est Pierre Bourdieu qui est à l'origine de ce concept et qui lui a donné sa première définition (1980). Cette notion s'est «imposée » à lui, dit-il, pour rendre compte « des effets où la sociologie spontanée reconnaît volontiers l'action des "relations" ».

Dans une contribution récente, Godechot et Mariot renouvellent l'approche du concept en soulignant que, dans la littérature, la divergence entre les approches existantes (Putnam, 1993; Coleman, 1988; Bourdieu, 1980; Burt, 1995) tient moins «aux différences de fondement de l'action (rejet ou adoption du paradigme de l'action rationnelle) qu'aux différentes conceptions des relations profitables» (2004, p. 244). Ils proposent ainsi de distinguer «deux formes de capital social»: l'une «individuelle», liée à la position de type broker (d'intermédiaire) d'un individu au sein d'une structure relationnelle', l'autre «collective», liée à la position de type closure (dense, cohésive) de plusieurs individus au sein d'une structure relationnelle ${ }^{2}$. Outre la question du «profit» qui est clairement posée, la problématique telle qu’elle est construite par Godechot et Mariot n’est pas étrangère aux préoccupations des économistes et des sociologues de l'économie, puisqu'il s'agit de la «concurrence»,

I Inspirée des travaux de Granovetter (1973) sur les «liens faibles» et de Burt (1995) sur les «trous structuraux».

2 Inspirée des travaux de Coleman (1988) sur la «fermeture» relationnelle. Mais qui renvoie aussi au concept de «niche sociale» chez Lazega (2009; avec Mounier, 2002), et aux plus récents travaux de Burt $(2005,2008)$. 
qui est ici posée comme un postulat de départ: «les deux formes de capital social peuvent être lues comme des modalités de gestion de la concurrence » (ibid. p. 246-247) ${ }^{3}$ au niveau des individus et des collectifs.

Dans une perspective proche, mais qui s'efforce de placer au même niveau concurrence et coopération, la théorie néo-structurale de Lazega (2009; avec Mounier, 2002) s'attache à décrire le «travail relationnel» (2009, p. 540) que les individus et les collectifs au travail, doivent fournir pour être en mesure d'accomplir leurs tâches, de réaliser leurs productions. Ce travail relationnel consiste en la circulation (sous forme de transferts et échanges) de ressources multiples et hétérogènes. Fidèle à une conception collective du capital social ${ }^{4}$, cette théorie insiste sur le concept de «niche sociale» (p.547) définie comme un «sousensemble de collègues-concurrents qui ont des relations spécialement denses, multifonctionnelles, durables » dans un milieu professionnel, au niveau intra- ou interorganisationnel (au sein d'une organisation ou au sein d'un marché). Mais, attachée à la figure d’un individu «stratégique», et soucieuse de contribuer à l'analyse de processus sociaux, tels le contrôle et la régulation, cette approche mobilise aussi la notion de «statut» dans une conception wébérienne (p. 548). De ce point de vue, les individus sont considérés comme inégaux, non pas seulement du fait des ressources potentielles dont ils sont susceptibles de disposer au travers de leur réseau (brokerage ou closure), mais aussi du fait de l'existence de ressources exogènes à leur réseau de relations et dont ils disposent en propre rejoignant ainsi la «théorie des ressources sociales» de Lin (1995) ${ }^{6}$, mais aussi la notion de «capital» chez Bourdieu (1992, p. 74 et suiv. ; 2000, p. 236 et suiv.).

3 Et aussi: «En concurrence pour des biens rares, des individus peuvent soit mobiliser individuellement les appuis efficaces pour l'obtention de ces biens, soit s'entendre avec certains concurrents, essayer de limiter la concurrence, former un groupe et mettre ce groupe en mouvement pour la monopolisation de ces biens rares».

4 Voir aussi Lazega, Lebeaux (1999) et Lazega (2005).

5 Dans une étude réalisée au sein un cabinet d'avocats d'affaires aux États-Unis, Lazega (1999, 200 I) cite, par exemple, l'existence de formes de statuts liés à la «contribution à la firme», l'«histoire des succès passés», les «compétences établies et reconnues», les «titres, qualifications, diplômes».

6 L'auteur y fait la distinction entre «les ressources personnelles et les ressources sociales. Les premières sont possédées par l'individu qui peut en disposer avec beaucoup de liberté. Les secondes 
Il nous semble, finalement, que l'examen des contributions sociologiques les plus intéressantes concernant la notion de capital social, nous ramène aux origines de celle-ci, c'est-à-dire à la définition qu’en avait donnée Bourdieu en 1980, dans des notes provisoires. Non seulement, comme l'indiquent Godechot et Mariot, chez Bourdieu, «le capital social est vu comme un démultiplicateur des autres capitaux (économique et culturel). [...] [Il] est comme le capital symbolique, un méta-capital» (ibid. p. 245); mais aussi, comme on le rappelle trop peu souvent, le capital social d'un individu ou d'un groupe est, inséparablement, une combinaison entre, d'une part, la configuration du «réseau de relations», et d'autre part, le «volume et le type de capital possédé en propre» (Bourdieu, 1980, p. 2). Cela tient au fait que Bourdieu fait la différence entre deux types de relations sociales «irréductibles»: d'un côté, le réseau de relations renvoie aux interactions, c'est-à-dire aux transferts et échanges de ressources, étudiés par la théorie néo-structurale; de l'autre, les «relations objectives» de proximité et d'opposition (liées au volume et à la structure du capital) qui existent entre individus ou groupes dans l'espace social ou dans le champ (sans que ceux-ci aient besoin d'être en interaction) renvoient à la hiérarchie sociale, c'est-à-dire aux types de statuts, étudiés par la théorie néo-structurale.

L’objet de cet article est, à partir d'un cas empirique, de faire le lien entre champ et réseau. Ce programme de recherche est, selon nous, contenu dans les notes provisoires de Pierre Bourdieu, mais ce dernier ne l'a jamais poursuivi. Parmi les raisons qui expliquent cela, il y a sans doute le fait qu'il était critique envers la network analysis ${ }^{7}$; mais aussi, le fait que puisque le capital social est «second par rapport aux autres formes de capitaux qu'il démultiplie», celui-ci «apparaîtrait ainsi redondant dans les analyses statistiques, surtout avec les

sont insérées (embedded) dans son réseau. Ce ne sont pas des biens que l'individu possède, mais des ressources accessibles au travers de ses liens directs et indirects» (p.687).

7 «Ce qui existe dans le monde social, ce sont des relations - non des interactions ou des liens intersubjectifs entre des agents, mais des relations objectives qui existent "indépendamment des consciences individuelles", comme disait Marx» (1992, p.72). «Dans la network analysis, [la structure de la distribution des ressources (ou des espèces de capital] a été sacrifiée à l'analyse des liaisons particulières (entre des agents ou des institutions) et des flux (d'information, de ressources, de services, etc.) dans lesquels elles se manifestent» (1992, p. 89). Voir aussi: 2000, p. 242. 
techniques comme l'analyse des correspondances ${ }^{8}$, plus synthétique qu'analytique » (Godechot et Mariot, op. cit., p. 245). De Nooy (2003) montre, en effet, que la mise en œuvre conjointe de l'analyse des correspondances et des outils d’analyse de réseaux sociaux (Wasserman et Faust, 1994 ; Lazega, 1998) permet d'aboutir à des résultats similaires.

Notre approche est différente dans la mesure où nous défendons plutôt l'idée que l'analyse de réseaux sociaux et l'analyse en termes de champ sont complémentaires plus que redondantes. Pour tenter de le montrer, notre article se fonde sur les données d'une enquête de terrain réalisée par questionnaire en face à face auprès de trois cents restaurateurs lillois, propriétaires indépendants de leur(s) établissement(s), auprès desquels nous avons recueilli trois types de variables différents: les attributs des restaurateurs eux-mêmes, les caractéristiques de leurs restaurants, et des données de type relationnel9 ${ }^{9}$. De par notre objet d'étude, à savoir des entreprises engagées sur un marché, notre contribution s'inscrit résolument dans la sociologie économique (Swedberg, 1994; Gislain, Steiner, 1995 ; Steiner, 1999; Lévesque, Bourque, Forgues, 200 I ; Convert et Heilbron, 2004; François, 2009). Une littérature au sein de laquelle nous constatons que, contrairement à l'analyse de réseaux sociaux, l'anthropologie économique de Pierre Bourdieu occupe une place relativement marginale. Avant de présenter nos résultats empiriques, nous tenterons de montrer en quoi celleci nous semble pourtant apporter une contribution essentielle (Boyer, 2003).

8 À savoir l'outil statistique sur lequel se base la construction empirique d'un champ chez Bourdieu.

9 Notre générateur de noms était: «Quels sont les restaurateurs que vous connaissez et qui vous connaissent aussi personnellement?». Suivaient différentes question sur le type de relation entretenu avec chacun des restaurateurs cités (connaissance, ami professionnel, ami personnel) et le type de ressources sociales échangé (discussions de conjoncture; informations intéressantes; renvois de clients; dépannages, coups de mains). 


\section{LE MARCHÉ COMME CHAMP}

Un marché suppose «une confrontation entre une/des offre(s) et une/ des demande(s) dans le but de procéder à des échanges sur la base d'un système de prix, qui résulte d'une négociation entre les parties en présence » (Guerrien, 2002, p. 320). Cette définition relativement consensuelle ne doit pas cacher les antagonismes disciplinaires auxquels la notion de marché donne lieu au sein des sciences sociales, dès lors qu'il s'agit de comprendre comment il fonctionne. En faisant le choix, dans cet article, de considérer le marché comme un champ, au sens de Pierre Bourdieu, nous assumons le fait de prendre un parti tranché dans un débat qui ne l'est pas. Considérer le marché comme un champ, c'est en effet prendre le contre-pied du paradigme dominant, celui de l'économie standard pour laquelle le marché est un «mécanisme abstrait» qui, moyennant la réalisation de certaines conditions (notamment de concurrence entre les individus), serait en mesure de produire un «équilibre» susceptible de correspondre «à un optimum du point de vue de la collectivité » (Boyer, 2009, p. 55). Alors qu'au fondement du «modèle de concurrence parfaite » (le plus usité), l'idée de concurrence est associée à l'idée d’une «égalité » (au moins au départ) des individus (Guerrien, 2002, p. 90), au fondement du concept de champ, on trouve, à l'inverse, l'idée d'une «inégalité » des individus, de sorte que «certains ont plus de pouvoir que d'autres » et que «la concurrence ne sert pas l'égalisation des chances, mais la reproduction d'une distribution inégalitaire du capital» (Boyer 2003, p. 69). Si l'hypothèse de «concurrence parfaite», les économistes eux-mêmes en conviennent ${ }^{10}$, n'est qu'un «cas idéal» et «ne peut être qu'irréaliste» (Guerrien, 2002, p. 89), c'est donc fondamentalement une question de méthode qui oppose les économistes standard et les sociologues de l'économie dont l'ambition est de «construire une définition réaliste de la raison économique» (Bourdieu, 2000, p. 235).

10 «Pour Friedman (...) les présupposés et les hypothèses des raisonnements n'ont pas besoin d'être réalistes» (François, 2009, p. 124). 
Outre qu'il implique dès le départ, entre les individus, une distribution inégalitaire des ressources actuelles (à travers la distribution inégale du capital économique et du capital culturel notamment) et potentielles (à travers les configurations différentes prises par le capital social), le champ implique aussi le fait que les individus qui y sont engagés

ont en commun un certain nombre d'intérêts fondamentaux, à savoir tout ce qui est lié à l'existence même du champ : de là une complicité objective qui est sous-jacente à tous les antagonismes. (...) Ceux qui participent à la lutte contribuent à la reproduction du jeu en contribuant (...) à produire la croyance dans la valeur des enjeux ${ }^{11}$. (Bourdieu, 1984, p. I15-116).

C'est ici une autre hypothèse, considérée comme fondamentale du point de vue de l'économie néo-classique, qui est une fois encore prise à contre-pied, à savoir «l'hypothèse d'atomicité» des individus qui suppose «que l'économie comporte un très grand nombre d'agents, et que ceux-ci sont de taille semblable (du point de vue de la production ou de la consommation), de sorte quaucun d'entre eux n'ait plus de poids que les autres» (Guerrien, 2002, p. 36). Cette hypothèse contribue à évacuer d'emblée de l’analyse économique, d'une part, la question de la coopération entre concurrents sur un marché, phénomène qui est assimilé à de la distorsion de concurrence, et d'autre part, la question des rapports sociaux qui sous-tendent les activités de production et d'échange économiques, et qui sont considérés moins sous l'angle de la confrontation que sous l'angle de la coordination entre les différentes parties prenantes, à travers un système de prix.

L'adoption de ces hypothèses peut s'expliquer par le fait que ce qui semble préoccuper les théoriciens néoclassiques c'est moins le fonctionnement d’une économie capitaliste que celui d’une économie de marchés, où le mot «marché » rassemble des objets aussi divers que les marchés de biens, le marché du travail ou les marchés financiers, et pour l’analyse desquels un modèle théorique unique (ou quasi) est proposé. Cette perspective «marché-centrique» devient contradictoire avec la perspective «capitaliste-centrique» dès lors que

II On retrouve cette idée chez White (198I, 2002): « les comportements des acteurs sont de nature concurrentielle, (...) il ne s'agit pas d'éliminer le concurrent car on est lié à lui pour le maintien de la structure du marché» (Steiner, 2005, p. 43). 
l'on aborde la question du profit ${ }^{12}$. L'économie néoclassique suppose que, dans le fonctionnement idéal d'une économie de marchés, «soit le profit est nul (en situation d'équilibre de concurrence parfaite) », «soit le profit n'est pas nul (parce qu'il est lié à l'existence d'une rente de situation)», «soit n'est pas nul, mais n’est positif que momentanément (car c'est un surprofit lié au déséquilibre momentané entre la demande et l'offre)»(Guerrien, 2002, p. 415-416). Cela tient au fait que, pour elle, les entreprises sont price-takers, elles subissent la loi du marché à travers la concurrence. Sur un marché conçu comme un champ, c'est une conception inverse de l'entreprise qui se déploie ${ }^{13}$ :

c'est la structure du champ, c'est-à-dire la structure du rapport de force (ou des relations de pouvoir) entre les entreprises, qui détermine les conditions dans lesquelles les agents sont amenés à décider (ou à négocier) les prix d’achat (de matériaux, du travail, etc.) et les prix de vente. (Bourdieu, 2000, p. 240).

Cette conception renvoie à la notion postkeynésienne d'entreprises price makers (Dallery, Éloire, Melmiès, 20ıo). Elle implique une analyse de la fixation des prix et de la réalisation du profit gouvernées par les rapports que chaque entreprise (en fonction de sa position dans le champ) est susceptible d'entretenir avec les différentes parties prenantes d'un marché (consommateurs, fournisseurs ${ }^{14}$ et pairs/concurrents) $)^{15}$, étant entendu que ces rapports, du fait que les intérêts des uns s'opposent aux intérêts des autres, sont mutuellement conflictuels (Dallery, van Treeck, 2008).

12 Chez Marx, il s'agit de «la plus-value produite par les travailleurs, que s'approprient les proriétaires des capitaux» (Guerrien, 2002, p. 415).

13 «La notion de champ marque la rupture avec la logique abstraite de la détermination automatique, mécanique et instantanée du prix sur des marchés livrés à une concurrence sans contrainte» (Bourdieu, 2000, p. 239-240).

I4 Ici, les «fournisseurs» ne comprennent pas seulement les entreprises sous-traitantes ou partenaires, ou les fournisseurs de matières premières mais aussi les fournisseurs de force de travail, c'est-à-dire les salariés.

15 Chez White (1981, 2002), la majorité des marchés réels sont, non pas bipartites (offre et demande) mais tripartites (consommateurs downstream, fournisseurs upstream, et producteurs interface). 
Ainsi, comme le rappelle Pierre Bourdieu, le champ n'est pas seulement «un champ de force mais aussi un champ de luttes» (Bourdieu, 2000, p. 244). C'est tout autant un lieu de concurrence au sens de Simmel, c'est-à-dire un lieu de conflits indirects entre des entreprises poursuivant les mêmes objectifs, qu'un lieu d’affrontements directs entre des groupes sociaux antagonistes et différentiellement dotés en pouvoirs de négociation. Nous avons proposé ailleurs (Dallery, Éloire, Melmiès, 2009) une illustration empirique de la capacité qu'ont les entreprises à réaliser du profit sur un marché en fonction de leur stratégie de profit (Boyer, Freyssenet, 200o). Dans cette étude, qui concerne aussi les restaurateurs lillois que nous évoquerons ci-après, nous établissons un lien entre le style de cuisine du restaurant et son taux de marge nette (le profit), de sorte que ce sont les restaurants qui font du «volume» (grande taille, petit prix) qui ont le taux de marge le plus élevé; et ceux qui font de la «qualité » (établissements gastronomiques) qui ont le taux de marge le plus faible voire négatif. Ces résultats, contre-intuitifs du point de vue de la théorie néoclassique ${ }^{16}$, montrent que les possibilités de profit attachées aux différentes positions dans la structure du champ sont inégales, et que cette inégalité provient de la capacité de négociation des entreprises avec les parties prenantes. Structuralement, les restaurants qui font du volume sont en meilleure position dans les négociations commerciales que les restaurants qui font de la qualité. Les premiers peuvent mettre la pression sur leurs salariés (faibles salaires, temps partiels, turnover élevé) et sur leurs fournisseurs (report sur eux de la concurrence en négociant des prix bas) et ainsi préserver leur marge. À l'inverse, les seconds ne peuvent réduire leurs coûts car «la qualité, ça se paye », autrement dit, ils doivent payer au prix fort leurs fournisseurs (pour avoir les meilleurs produits) et leur personnel (pour qu’il soit qualifié et stable).

16 Contre-intuitifs, parce que du fait des petits prix pratiqués par les restaurateurs qui font du volume, la concurrence devrait être plus importante entre eux, et donc les marges plus faibles (du fait de cette concurrence); et à l'inverse, du fait de la singularité des établissements gastronomique, ceux-ci devraient se trouver sur des niches où les effets de la concurrence se font moins sentir, donc où les marges peuvent être plus élevées. 
Ces analyses contredisent, selon nous, l'hypothèse de l'homo œconomicus, laquelle postule que tous les individus, a fortiori les chefs d'entreprises, cherchent en permanence à maximiser leur profit. Si tel était le cas, la cuisine gastronomique aurait fini par disparaître du fait de son manque de rentabilité en comparaison d’autres styles culinaires. Si dans la réalité, elle subsiste, c'est aussi parce que, dans le monde économique, comme dans le monde social, il existe différentes formes d’intérêt et de statut, qui sont irréductibles à l'intérêt économique. Outre le statut économique, nous nous intéresserons ici au statut gastronomique, dont le caractère symbolique est tout autant objet, de la part des restaurateurs qui souhaitent l'atteindre, de stratégies de gestion et d'investissement. Ces stratégies prennent un caractère spécifique qui n'est plus celui d’un calcul rationnel visant «la maximisation d'un produit et la minimisation des coûts» (Castoriadis, 1999, p. 8I), mais celui d’un «travail relationnel» dont nous pensons qu'il peut être décrit au travers de la notion de capital social, prise dans sa définition originelle.

\section{CAPITAL SOCIAL, NICHES SOCIALES ET STATUT}

«Le capital social est l'ensemble des ressources mobilisées à travers un réseau de relations plus ou moins étendu et plus ou moins mobilisable qui procure un avantage compétitif en assurant aux investissements des rendements plus élevés ». Cette définition, dans laquelle Pierre Bourdieu reformule (2000, p. 237), vingt ans après, ce qu'il avait énoncé dans ses notes provisoires, est assortie d'un commentaire qui vise à la distinguer «des définitions qui ont été avancées ensuite dans la sociologie et l'économie américaines». Si le point commun à toutes les définitions du capital social est le caractère instrumental de ce concept, c'est-à-dire le fait qu'il vise toujours à modéliser, à expliquer la performance, l'efficacité, la profitabilité de l'objet d'étude auquel il s'applique, la conception de Pierre Bourdieu possède, selon nous, plusieurs particularités. Une première particularité est, comme nous l'avons évoqué au début de cet article, le fait qu'elle s'efforce d'intégrer deux manières de concevoir les relations sociales, à la fois comme «interactions sociales» et comme «structures sociales» (indépendantes des interactions). Une deuxième particularité est qu'elle se donne les moyens d'intégrer, de façon non normative, les deux 
formes de capital social qui ont été repérées dans la littérature par Godechot et Mariot: peu importe, en quelque sorte, la manière dont le capital social est bénéfique, l'essentiel ici est qu'il le soit. Une troisième particularité est qu'elle ne nous semble pas vouloir choisir entre efficacité pour l'individu et efficacité pour le collectif. Outre son caractère instrumental, le capital social apparaît aussi comme un principe conservateur puisqu'il participe, fondamentalement, à la reproduction de l'ordre social (ou économique), à la démultiplication des avantages de certains (au détriment d'autres), donc à la reproduction des inégalités en général, et ce, que ce soit au niveau individuel ou au niveau collectif. Mais qu'entend-on ici par «niveau collectif»? Est-ce à dire que le capital social peut être simultanément un concept porteur d’inégalités et de solidarité ? Oui, à condition d'entendre la notion de solidarité non pas dans sa conception universaliste mais dans une conception particulariste, c'est-à-dire s'appliquant à un groupe restreint, à un collectif dont les limites, ou frontières, sont tangibles.

La littérature offre des exemples d'études du capital social «collectif». Coleman prend l'exemple des «diamantaires de New York, où le haut degré de confiance entre marchands leur permet de s'échanger sans formalité pour expertise des diamants de très grande valeur» (Ponthieu, 2006, p. I3). Chez Putnam, c'est au niveau agrégé d’une région, d'un État ou d'une nation que le capital social «s'accumule» (à travers l'engagement civique et associatif des citoyens par exemple), ce qui conduit à «de meilleures performances sociales, politiques ou économiques» (Ponthieu, 2006, p. 43-44). Chez Bourdieu (2007, p. 386), c'est par exemple la «noblesse», groupe social caractérisé par «la possession, l'entretien, la reproduction» d'un type particulier de capital social, «châteaux, terres, etc. », mais aussi «connections» à travers les jeux de cricket, de rugby, etc.

Basée sur l'analyse des réseaux d'échanges de ressources sociales, la théorie néo-structurale propose une autre façon de concevoir les «collectifs» porteurs de capital social. Au sein d'une population, elle s'efforce de repérer endogènement des sous-ensembles d'acteurs, appelés niches sociales, à partir de la connaissance empirique des interactions que les membres de cette population entretiennent entre eux. Le critère individuel d'appartenance à une niche sociale n'est pas, ici, lié à un attribut ex-ante (être diamantaire, être citoyen 
d'un État, être noble), mais est typiquement relationnel : l'individu appartient à une niche sociale s'il est en interaction avec d'autres individus de la niche. Plus les membres d'une niche sont en interrelation, plus celle-ci apparaît cohésive (ou dense). La théorie néo-structurale interprète l'existence, au sein d'une population, d'un système de niches sociales, comme une manifestation du processus de solidarité particulariste évoqué plus haut. Le repérage des niches sociales constitue donc une contribution à l’analyse du capital social individuel et collectif. Les individus coopèrent au sein des niches sociales, la règle étant de suspendre (temporairement et conjoncturellement) le comportement calculateur pour le bien de ces «collectifs » informellement constitués. Il n’y a donc jamais de déconnection entre les intérêts individuels et les intérêts collectifs, car les individus ont un intérêt fonctionnel et instrumental à la construction des niches sociales, au sein desquelles ils trouvent les ressources sociales qui leur sont nécessaires (information, entraide, conseil, etc.). La théorie néostructurale propose des exemples empiriques sur des objets à la fois intra- et interorganisationnels (organisations et marchés; voir Lazega, 2009).

Une fois repéré le système de niches et les individus qui les composent, il est intéressant de revenir aux attributs sociaux (ex-ante) de ces derniers afin de comprendre les principes qui guident la construction des niches sociales : a priori, on n'interagit pas avec n'importe-qui. Cette étape de l'analyse est d'autant plus importante que nous nous situons dans le cadre d'un marché-champ fondé à la fois sur l'interdépendance et l'inégalité entre agents sociaux. L’hypothèse que nous posons, et qui est poursuivie par la théorie néo-structurale, est que les niches sociales sont non seulement le lieu de la production et de la reproduction du capital social individuel et collectif, mais aussi de la «concurrence de statut » à laquelle se livrent les agents du milieu étudié. Ainsi, selon la théorie néo-structurale, avec la construction de niches sociales, la concurrence de statut constitue un autre phénomène important de la vie sociale et économique. Telle que nous la concevons ici, la «concurrence» ne se réduit pas au processus concurrentiel des économistes néoclassiques. On trouve, chez Simmel, on l’a dit, une définition générique de la concurrence comme «forme particulière de lutte» (François, 2009, p. 237). Particulière, parce qu'il s'agit 
d'une lutte indirecte dont les caractéristiques sont les suivantes : une lutte entre des acteurs I) ayant conscience d'être en lutte, 2) réalisant des efforts parallèles en vue d'atteindre un but, 3) et dont le résultat sera que si l'un gagne, l'autre perd (critère qui la différencie donc de l'émulation). Quant au concept de statut, dans une perspective structurale, il prend un caractère composite, du fait de l'existence de multiples formes de capital : économique (basé sur le contrôle de l'appareil de production et sur le revenu), culturel et symbolique (éducation, honneur, prestige, réputation), ou politique (contrôle de l'appareil d'État). Et, dans une perspective relationnelle, le statut se caractérise par la concentration et l'accumulation de ressources sociales. La possession simultanée, par un même individu, de plusieurs types de statut différents est ainsi à la base de la fonction de leadership : on parle alors «d'oligarque multi-statutaire» (Lazega, 2003, p. 320-32I). Dans ce processus de concurrence visant l'accès aux différentes formes de statut, le capital social joue, selon nous, un rôle essentiel. Ce que nous souhaitons illustrer au travers d'une étude de cas empirique, celle d'un champ gastronomique à l'échelle locale.

L'enquête de terrain et le questionnaire

Les données sur lesquelles nous nous fondons sont issues d'une enquête de terrain réalisée (entre février et juillet 2006) par questionnaire en face à face, auprès d'environ trois cents restaurateurs de la métropole lilloise. Construit sur la base d'une préenquête qualitative (dix entretiens semi-directifs réalisés entre avril et octobre 2005), notre questionnaire comporte trois types de variables: premièrement, les attributs du restaurateur (âge, ancienneté, expérience, diplôme, récompenses, etc.); deuxièmement, les caractéristiques de son/ses établissement(s) (taille, style de cuisine, prix pratiqués, nombre de salariés, etc.); troisièmement, les relations de transferts et échanges de ressources sociales entre restaurateurs (discussions de conjoncture, informations intéressantes, etc.) dont nous avons identifié l'existence lors de notre pré-enquête.

\section{La construction de la population}

Pour circonscrire empiriquement notre population de restaurateurs, nous nous sommes appuyés sur différents critères, faisant en sorte que les enquêtés répondent simultanément à plusieurs de ces critères (mais pas nécessairement à tous en même temps): être situé sur la métropole lilloise; tenir un restaurant de type 
traditionnel (au sens de la $\mathrm{NAF}^{17}$, qui distingue restauration rapide et traditionnelle); être indépendant juridiquement (ce qui exclue donc les chaînes et les franchises); participer au réseau de transfert et d'échange des différentes ressources sociales que nous avons identifiées; appartenir institutionnellement au milieu professionnel (en étant membre d'une association ou d'un syndicat, en possédant une récompense honorifique dans des guides gastronomiques, etc.).

\section{Le statut de l'échantillon}

Ainsi construite sur une diversité de critères, notre population n'a pas vocation à être un échantillon représentatif, mais à constituer un «échantillon théorique» (Glaser et Strauss, 1967) dont l'intérêt ne réside pas dans sa capacité à décrire une population plus large, mais à tendre vers le maximum de diversification des données, donc des résultats. La logique de construction d'un champ appartient selon nous à ce type d'échantillon.

\section{La construction du champ}

Pour construire les volets «attributs des restaurateurs» et «caractéristiques des établissements» de notre questionnaire, nous avons utilisé la démarche en termes de «champ» proposée par Bourdieu (1992, p. 196 et suiv.). Cet outil permet de représenter un espace social sous ses différentes dimensions (sociale, économique, culturelle, symbolique) et d'en saisir les principes de division objectifs. II se fonde sur un type d'analyse statistique, l'analyse des correspondances multiples (ACM) entre un ensemble de variables considérées comme pertinentes du point de vue de l'objet étudié. Tout l'enjeu étant de définir ces «variables pertinentes»: pour ce faire, nous nous sommes référés à d'autres analyses empiriques et avons cherché à raisonner par analogies. Nous avons remarqué que, si certains champs ont pour unité d'analyse des individus: c'est le cas du «champ des avant-gardes artistiques» (Verger, 1991), du «champ littéraire français sous l'Occupation» (Sapiro, 1996), du «champ des économistes français» (Lebaron, 1997); d'autres champs ont pour unité d'analyse des organisations: c'est le cas du «champ du journalisme économique» où les unités sont des médias (Duval, 2000), du «champ de l'édition française» (Bourdieu, 1999), du «champ de la haute couture» (Bourdieu, Delsaut, 1975). Ce second cas de figure est intéressant car il est proche du notre: il s'agit, en effet, de coupler des données qui s'attachent aux individus à des données qui s'attachent aux organisations ${ }^{18}$. Dans ces

17 Nomenclature des activités françaises.

I8 Une adaptation de cette approche multi-niveaux dans le cadre la théorie néo-structurale est contenue dans la mise en œuvre de la méthode dite du linked design, qui consiste à examiner séparément des réseaux «complets» de niveaux différents, constituant deux systèmes d'interdépendances superposés, partiellement emboîtés, l'un interorganisationnel, l'autre interindividuel (Lazega, Jourda, Mounier, Stofer, 2007). 
différentes études, les attributs sont classés par grandes familles: certaines variables renvoient directement aux caractéristiques des individus et à leurs dispositions (sexe, âge, origine familiale, trajectoire scolaire, diplôme); d'autres sont attachées aux organisations, aux entreprises plutôt qu'aux individus, il s'agit de variables de positionnement économique (origine du fond, statut juridique, capital social, nombre de salariés, chiffre d'affaires, horaires d'ouverture, fréquentation, répartition interne des rôles, nombre de couverts, prix pratiqués, publicité); enfin, d'autres variables dites de position ou de prise de position, sont plus spécifiques et liées à chaque objet d'étude: il s'agit ici de l'activité de restaurateur et de son organisation en termes de champ, non seulement économique, mais aussi gastronomique (ancienneté dans le métier, parcours et trajectoire professionnelle et scolaire, diplôme possédé, fonctions syndicales, mais aussi récompenses, distinctions, citations dans les guides, style

de cuisine) À travers ce canevas, nous possédons le fond et la structure d'ensemble des parties I et 2 de notre questionnaire: la forme et l'ordre des questions étant établis à la suite de tests et de nombreux tâtonnements.

\section{La reconstitution des réseaux sociaux}

Le volet «sociométrique» (partie 3 du questionnaire) est ce qui fait la spécificité de notre approche en termes d'analyse de réseau. Alors que les attributs permettent de construire le champ des relations objectives qui s'établissent entre les restaurateurs; les questions sociométriques sur les choix des partenaires et les échanges de ressources permettent de reconstituer la structure relationnelle. Sur la base du générateur de noms suivant: «quels sont les restaurateurs que vous connaissez personnellement et qui vous connaissent personnellement aussi évidemment?», les restaurateurs enquêtés sont amenés à dresser la liste des collègues/concurrents avec lesquels ils sont en contact. Cette question est large et ne vise qu'à générer une liste de noms (non limitée dans l'absolu). Sa formulation fait ressortir consciemment et explicitement la notion de réciprocité: il ne s'agit pas de citer des personnes «connues» (au sens médiatique, par exemple) mais bien des personnes avec lesquelles l'enquêté est susceptible d'avoir des interactions. Une fois la liste de contacts établie, il s'agit de connaître le type de lien entretenu avec chaque personne citée: l'ancienneté de l'interconnaissance, les liens familiaux ou amicaux, les échanges de ressources: discussions de conjoncture; informations intéressantes; conseils; dépannages; renvois de clients. L'enquête sociométrique fait appel à la mémoire des enquêtés et évidemment à leur subjectivité: le questionnaire enregistre la manière dont les individus se perçoivent les uns par rapport aux autres. Selon la méthodologie que nous mettons en œuvre (Lazega, 1998), un réseau égale une ressource. C'est la superposition des différents réseaux d'échanges de ressources qui traduit la «multiplexité» des relations. 


\section{LA CONSTRUCTION DU CHAMP GASTRONOMIQUE}

La restauration traditionnelle est un commerce, mais c'est aussi un savoirfaire, ce qui implique un encastrement des dimensions économique et gastronomique. Nous avons décrit ailleurs la dimension économique du marché de la restauration lilloise (Dallery, Éloire, Melmiès, 2009). À travers le concept sociologique d'interface de marché (White, 1981, 2002), nous avons montré l'importance de la structure des coûts, de la taille des établissements et des prix pratiqués par eux dans la détermination de la structure économique du marché. Nous y avons souligné l'existence d'une concurrence partitionnée : des entreprises qui cherchent à faire du volume (des prix bas mais beaucoup de vente) ; des entreprises qui cherchent à faire de la qualité (des prix élevés mais des ventes plus modestes) ; entre les deux, une catégorie d'entreprises intermédiaires. Avec l'analyse en termes de champ, nous montrons les ressorts sociologiques qui sous-tendent cette structure économique. Les choix stratégiques des restaurateurs, d'orienter leur production plutôt vers le volume ou vers la qualité, dépendent d'attributs sociologiques, et notamment du capital culturel spécifique à la restauration, que nous avons appelé capital culinaire. Le capital culinaire est en quelque sorte le «bagage» accumulé par le restaurateur avant de s'installer à son compte: son diplôme et son expérience professionnelle dans le métier. Il se structure autour de trois pôles, correspondant à trois types de dispositions ou d'habitus de restaurateurs: les «autodidactes», propriétaires d'établissements de restauration sans diplôme hôtelier ni expérience de salarié dans ce secteur, que rien ne semblait donc prédisposer à tenir un restaurant plutôt qu'une autre activité $^{19}$, et qui apprennent donc le métier sur le tas; les «techniciens » qui ont un diplôme et/ou une expérience de salarié, plutôt en salle qu’en cuisine, plutôt courte, plutôt locale que nationale, et qui mettent en application un savoir-faire ; et les «artistes», qui sont comme les «techniciens», mais dont l'expérience est plutôt nationale, plutôt longue, plutôt polyvalente (salle et cuisine) mais aussi plutôt prestigieuse, c'est-à-dire acquise aux côtés de Chefs d'établissements haut 
de gamme, reconnus par les Guides gastronomiques, et qui usent de ce savoirfaire comme d'un moyen d'expression singulier.

Si ce capital culinaire joue un rôle structurant au sein du champ gastronomique (voir figure I), c'est qu'il contribue à l'existence d'une double hiérarchie entre d'une part, sur l'axe horizontal du champ, les «possesseurs » (capital culinaire+) et les «non-possesseurs» (capital culinaire-) de capital culinaire, et entre d'autre part, sur l'axe vertical, les «bien» (vers le haut) et les «mal» (vers le bas) dotés en capital culinaire. L'élément essentiel concerne l'opposition qui s'établit entre détenteurs de capital culinaire (à droite) et détenteurs de capital économique, à gauche), ce dernier étant mesuré par la taille de l'établissement (nombre de couverts, de salariés) et le volume de son chiffre d'affaires. Ainsi, l’axe horizontal présente une «structure en chiasme», qui renvoie d'ailleurs à la structure de «l'espace social global» repérée par Pierre Bourdieu ${ }^{20}$, de sorte que plus le capital culinaire est élevé, plus le capital économique est faible, et vice-versa. À droite de l’axe, du côté des restaurateurs «possesseurs» de capital culinaire, la distribution est inégale (c'est ce que résume notre distinction entre les «techniciens», en bas, et les «artistes», en haut de l'axe vertical). Cette distinction se manifeste par la possession, par les seconds, de capital gastronomique, ce dernier étant mesuré par la «note de qualité » des établissements ${ }^{21}$ et le degré de participation de leurs propriétaires à des associations «honorifiques » au sein desquelles ces Chefs se regroupent et se cooptent. À la différence du capital culinaire, le capital gastronomique est une reconnaissance de leur savoir-faire que les restaurateurs obtiennent après leur installation à leur compte. Les dispositions des restaurateurs, liées à leur capital économique et à leur capital culinaire, impliquent, de leur part, des prises de positions spécifiques en matière de choix du «style de cuisine»

20 «La structure de la distribution du capital économique est symétrique et inverse de la structure de la distribution du capital culturel» (1979, p. 128 et suiv.; voir aussi Bourdieu, 1994, p. 20-22).

21 Le calcul de la «note de qualité» de chaque restaurant s'effectue à partir des référencements et des distinctions décernées par les cinq principaux guides nationaux reconnus dans la profession, à savoir: Guide Michelin, Bottin Gourmand, Pudlo, Champerard, Gault \& Millau. Dans notre population, $83,5 \%$ des établissements n'ont pas de note (pas de distinction) et pour ceux qui en ont les notes s'étalent de I (simple citation) à 78 (beaucoup de citations et de récompenses). 
pratiqué $^{22}$ (voir la figure I, où les «styles de cuisine »-en italique - sont projetés en variable supplémentaire dans l’ACM). L'ordonnancement et la hiérarchie de ces styles entrent en cohérence avec l'agencement des formes de capital tel que nous l’avons décrit et qui peut être résumé ainsi : l’axe horizontal représente la structure du capital des restaurateurs et est marqué par l'opposition entre détenteurs de capital culinaire (à droite du plan) et détenteurs de capital économique (à gauche du plan); et l’axe vertical représente le volume de capital global des restaurateurs et est marqué par l'opposition entre bien dotés en capital de toute sorte (en haut du plan) et les mal dotés (en bas du plan).

Figure I : Le champ gastronomique

\section{Volume de capital global}

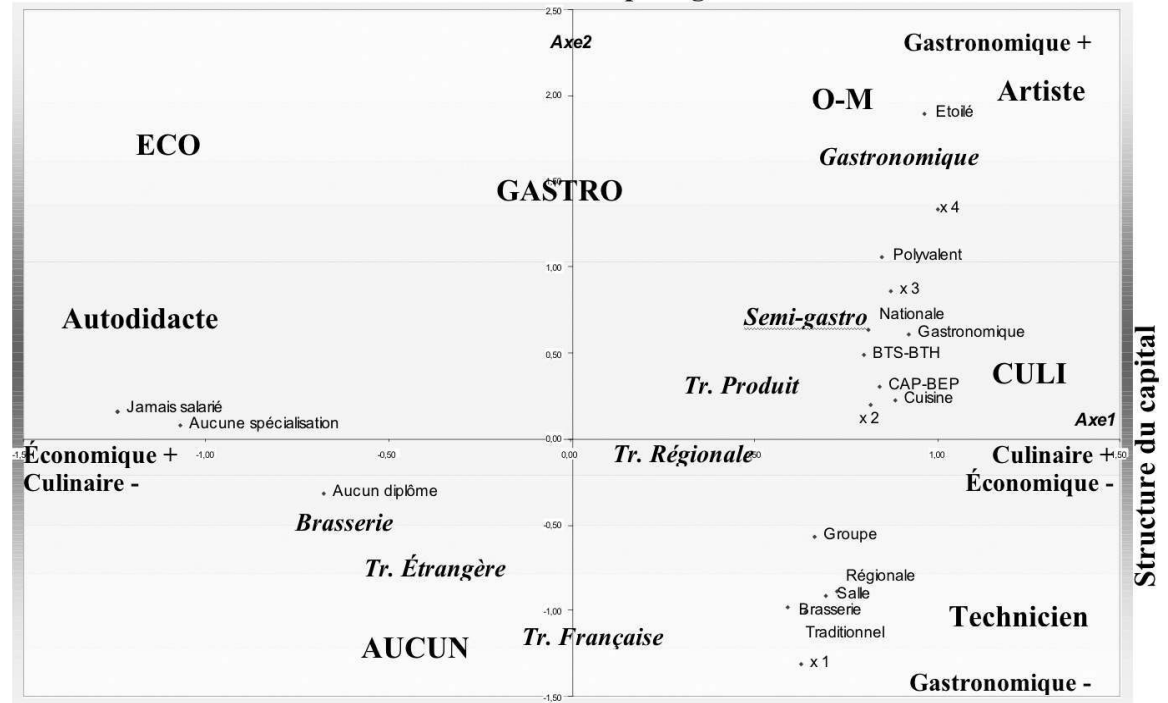

Source: Éloire, 2008.

22 La cuisine gastronomique (c'est-à-dire haut de gamme) se décompose en cuisine gastronomique et cuisine semi-gastronomique. La cuisine traditionnelle (c'est-à-dire la cuisine de moyenne gamme) regroupe des spécialités très diverses, que nous avons regroupées comme suit : cuisine traditionnelle française (tous types de plats et de recettes françaises); cuisine traditionnelle régionale (plats à connotation régionale); cuisine traditionnelle produit (cuisine spécialisée sur un produit ou sur un concept particulier) ; cuisine traditionnelle étrangère (pizzeria, couscous, etc.). La cuisine de brasserie (plutôt bas de gamme) désigne les établissements qui font à la fois café et plat du jour (le midi). 


\section{LE REPÉRAGE DES FORMES DE STATUT}

De chacune de ces formes de capital découle une manière différente d'être important au sein du marché-champ. Chez Bourdieu (1984, p. II3), «la lutte entre prétendants et dominants » est un «mécanisme générique », dont il faut chaque fois «rechercher les formes spécifiques, entre le nouvel entrant qui essaie de faire sauter les verrous du droit d'entrée et le dominant qui essaie de défendre le monopole et d'exclure la concurrence ». Ce qui fait écho, chez Lazega (2003, p. 318), à la «concurrence de statut» qui «permet aux acteurs de concentrer les ressources sociales pour se mettre en position de définir les termes de leurs échanges », c'est-à-dire en quelque sorte d'accumuler du capital social individuel; «mais aussi de faire converger vers les acteurs «importants » du système des ressources et des attentes », c'est-à-dire de forger une «discipline sociale» qui peut être comprise comme une forme de capital social collectif. Le repérage des individus possédant du statut se fonde sur les données liées au poids économique pour le capital économique (ÉCO), la note de qualité pour le capital gastronomique (GASTRO), et sur la typologie (autodidacte, technicien, artiste) pour le capital culinaire (CULI). Ce travail empirique nous conduit à repérer au sein de notre population : $38 \%$ de restaurateurs disposant d'une forme de statut quelle qu'elle soit (ce sont les oligarques) et $15 \%$ disposant de deux formes de statut au moins (ce sont les oligarques multi-statutaires - O-M). $47 \%$ de restaurateurs ne disposent donc d'aucun statut (voir le tableau I). Parmi les oligarques (une seule forme de statut), 69\% disposent uniquement de capital culinaire ; $18 \%$, uniquement de capital gastronomique; I4\%, uniquement de capital économique. 
Tableau I : Répartition des restaurateurs en fonction des formes de statut

\begin{tabular}{|c|c|c|}
\hline & EFFECTIFS & $\%$ \\
\hline AUCUN STATUT (AUCUN) & 133 & 47 \\
\hline OLIGARQUES & 107 & 38 \\
\hline Économique (ÉCO) & 15 & 14 \\
\hline Gastronomique (GASTRO) & 19 & 18 \\
\hline Culinaire (CULI) & 74 & 69 \\
\hline OLIGARQUES MULTI-STATUTAIRES (O-M) & 43 & 15 \\
\hline Éco + Culi & 8 & 3 \\
\hline Éco + Gastro & 6 & 2 \\
\hline Gastro + Culi & 26 & 9 \\
\hline Gastro+Culi+Éco & 3 & I \\
\hline TOTAL & 283 & 100 \\
\hline
\end{tabular}

Source: Éloire, Fagnoni, 2008.

Une analyse des corrélations entre les différentes formes de statut fait apparaître les congruences et non- congruences entre elles (voir le tableau 2) : le capital économique et le capital culinaire, sans avoir tendance à s'exclure mutuellement, ne sont pas corrélés. Par contre, le capital économique et le capital gastronomique sont positivement corrélés donc congruents, et il en va de même entre le capital culinaire et le capital gastronomique. Lorsque l'on s'intéresse aux oligarques multi-statutaires, on constate que toutes les formes de capital sont positivement corrélées entre elles, et donc contribuent à la construction de cette catégorie d'individus importants. Comme le montre le tableau précédent qui détaille les formes constatées d'oligarques multi-statutaires, les plus fréquentes sont celles qui allient des formes de statut congruentes (gastronomique et culinaire, notamment), les moins fréquentes sont celles qui allient des formes de statut non-congruentes (économique et culinaire). Lorsqu'on projette le statut en variable supplémentaire dans l'ACM (voir la figure I), on constate que toutes ses modalités sont situées au-dessus de l'axe horizontal. Les oligarques multi-statutaires $(\mathrm{O}-\mathrm{M})$ sont en haut à droite, 
du côté du capital gastronomique+. À l'opposé, les restaurateurs de statut économique (ÉCO) sont en haut à gauche, du côté du capital économique+. Ce qui distingue les oligarques multi-statutaires des restaurateurs disposant uniquement de statut culinaire (CULI), situés à droite mais au centre du plan, c'est moins la structure du capital que le volume global de capital possédé. À l'inverse, ce qui distingue les oligarques multi-statutaires des restaurateurs disposant uniquement de statut gastronomique (GASTRO), situés en haut mais au centre du plan, c'est moins le volume global que la structure du capital, à mi-chemin entre les pôles gastronomique+ et économique+. Les restaurateurs ne disposant d’aucun statut (AUCUN) sont situés en bas du plan.

Tableau 2 : Congruence et non-congruence entre les formes de statut

\begin{tabular}{|c|c|c|c|c|}
\hline Statuts & ECO & GASTRO & CULI & O. M-S. \\
\hline ECO & - & & & \\
\hline GASTRO & $\begin{array}{c}0,12 \\
(0,03)\end{array}$ & - & & \\
\hline CULI & $\begin{array}{c}-0,06 \\
(0,24)\end{array}$ & $\begin{array}{c}0,13 \\
(0,02)\end{array}$ & - & \\
\hline O. M-S. & $\begin{array}{c}0,39 \\
(<.0001)\end{array}$ & $\begin{array}{c}0,70 \\
(<.0001)\end{array}$ & $\begin{array}{c}0,37 \\
(<.0001)\end{array}$ & - \\
\hline
\end{tabular}

Source: Éloire, 2008.

Nous nous intéressons à présent au lien qui s'établit entre cette analyse du champ gastronomique et l'analyse des réseaux sociaux. Notre but est de donner à voir les différentes formes de «travail relationnel» que les restaurateurs effectuent en fonction de la position qu'ils occupent dans la concurrence de statut. L'hypothèse que nous posons est que la forme prise par le capital social varie selon que l'on se situe au pôle économique ou au pôle gastronomique du champ. L’analyse de réseau est mobilisée ici comme un outil. Trois types d’analyses sont ici menés, qui dévoilent la double facette du capital social (individuel et collectif) conformément à la définition originelle de ce concept. 


\section{CONCENTRATION DES RESSOURCES SOCIALES}

Le premier type d’analyse que nous présentons est celui des centralités. Dans le domaine de l'analyse de réseau, la mesure de centralité renvoie à une mesure individuelle de prestige. Il y a différentes manières d'être central au sein d'un réseau : être beaucoup cité par les autres membres du réseau (indegree), citer beaucoup d’autres membres du réseau (outdegree), être proche de beaucoup d'autres membres du réseau (closeness), être souvent en position d'intermédiaire entre les autres membres du réseau (betweeness). Ces mesures peuvent être calculées pour chaque individu. Nous nous demandons, ici, si en fonction du type de statut possédé, la centralité varie (voir le tableau 3). Effectivement, si l'on examine les scores de centralité dans le réseau d'échange d'informations intéressantes, on constate que les oligarques multi-statutaires tendent à concentrer vers eux cette ressource sociale ${ }^{23}$ : leur score d'indegree, par exemple (mais c'est aussi le cas pour les autres mesures) est significativement plus élevé que la moyenne (6,3 contre 2,6 dans l'ensemble du réseau d'informations). Ces restaurateurs s'avèrent donc être les plus investis dans le «travail relationnel». Dans le tableau, nous faisons la distinction entre deux profils d'oligarques multi-statutaires: ceux qui cumulent au moins les deux types de statut congruents, gastronomique et culinaire; et ceux qui possèdent au moins le statut économique (tendanciellement non-congruent avec le statut culinaire). Nous constatons que les premiers contribuent plus encore que les seconds à l'échange de ressources sociales $(8,2$ contre 2,8$)$. Par ailleurs, les restaurateurs ne disposant que du seul statut gastronomique possèdent aussi, en moyenne, un indegree élevé $(4,3)$. Ce qui n'est pas du tout le cas des restaurateurs possédant le statut économique ou le statut culinaire (respectivement I,I et I,6 contre 2,6 en moyenne sur l'ensemble du réseau). Le groupe des restaurateurs ne disposant d’aucun statut possède même des scores légèrement plus élevés que ceux de ces derniers $(\mathrm{I}, 8)$. Ce sont les restaurateurs disposant d'un statut uniquement économique qui savèrent, d’après les moyennes, les moins investis au sein des réseaux sociaux. Quant aux restaura-

23 Les analyses ont aussi été effectuées sur les réseaux de discussions de conjoncture, de dépannages, coups de mains et de renvois de clients: elles révèlent des tendances identiques. 
teurs disposant d'un statut uniquement culinaire, leur comportement relationnel est relativement similaire à celui de ceux qui ne possèdent aucun statut.

Tableau 3 : La concentration et le contrôle des ressources sociales par les oligarques multi-statutaires au sein du réseau d'informations

\begin{tabular}{|c|c|c|c|c|}
\hline & INDEGREE & OUTDEGREE & CLOSENESS & BETWEENESS \\
\hline $\begin{array}{lr}\text { O. MULTI-STAT. } & \\
\text { dont: } & \text { Éco } \\
& \text { Gastro-Culi }\end{array}$ & $\begin{array}{l}6,3 \\
2,8 \\
8,2\end{array}$ & $\begin{array}{l}6,0 \\
3,0 \\
7,6\end{array}$ & $\begin{array}{l}0,28 \\
0,22 \\
0,31\end{array}$ & $\begin{array}{c}0,01 \\
0,007 \\
0,02\end{array}$ \\
\hline ST. GASTRONOMIQUE & 4,3 & 3,2 & 0,26 & 0,007 \\
\hline ST. CULINAIRE & 1,6 & ।,8 & 0,20 & 0,002 \\
\hline ST. ÉCONOMIQUE & $I, I$ & 0,4 & 0,13 & 0,0002 \\
\hline AUCUN STATUT & 1,8 & 2,0 & 0,25 & 0,003 \\
\hline ENSEMBLE & 2,6 & 2,6 & 0,20 & 0,007 \\
\hline
\end{tabular}

Source: Éloire, 2008.

Ces chiffres montrent que la centralité dans les réseaux d'échange de ressources constitue un élément de discrimination essentiel entre les restaurateurs engagés dans la concurrence de statut gastronomique : lorsque la possession de savoir-faire (capital culinaire) se reconvertit, chez certains restaurateurs, en reconnaissance des pairs (capital gastronomique), c'est bien du côté du capital social comme «méta-capital», qu’il faut chercher l'explication.

\section{CONTRÔLE DES RESSOURCES PAR L'HOMOPHILIE DE STATUT}

Le deuxième type d'analyse que nous proposons est celui de l'homophilie de statut. Si le travail relationnel des restaurateurs de statut gastronomique est de concentrer les ressources sociales, nous faisons l'hypothèse que le contrôle de ces ressources passe par des phénomènes d'homophilie, c'est-à-dire par le fait qu'ils se recherchent mutuellement entre eux comme partenaires d'échange. Pour montrer cela, nous mettons en œuvre des modèles statistiques de type ERGM, qui testent la probabilité et la significativité d’apparition de certaines sous-structures dans le réseau. Nous en testons deux. La première sous-structure à laquelle nous nous intéressons est dyadique: elle teste la probabilité que, lorsqu'un lien existe entre deux 
restaurateurs, ces deux restaurateurs partagent le même attribut. La seconde sousstructure est triadique: elle teste la probabilité que, lorsqu'un cycle ${ }^{24}$ existe entre trois restaurateurs, ces trois restaurateurs partagent le même attribut. L’attribut testé dans notre modèle est le type de statut possédé (voir le tableau 4). Les résultats indiquent la présence de d'homophilie (dyadique et triadique) chez les oligarques multi-statutaires et chez les restaurateurs de statut gastronomique, ce qui vient confirmer notre hypothèse selon laquelle il s'effectue, à ce pôle du champ, un travail de concentration et de contrôle des ressources sociales.

Tableau 4: Les formes de statut comme signaux de reconnaissance au sein du réseau d'informations

\begin{tabular}{|c|c|c|}
\hline Paramètre (écart-type) & HOMOPHILIE DYADIQUE & ÉCHANGE GÉNÉRALISÉ \\
\hline OLIGARQUES MULTI-STATUTAIRES & $+0,508 \mathrm{I}(0,0004)^{* * * * *}$ & 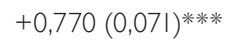 \\
\hline STATUT CULINAIRE & $+0,699(0,0003)^{* * * *}$ & $-0,60$ I $(0,029)^{* * * *}$ \\
\hline STATUT ÉCONOMIQUE & $-|0,9|(0,003)^{* * * *}$ & $-0,68(0,016)^{* * * * *}$ \\
\hline STATUT GASTRONOMIQUE & $+0,15(0,0002)$ ***** & $+0,018(0,054)^{* * * * * *}$ \\
\hline
\end{tabular}

Source: Éloire, 2007; Logiciel Statnet.

$\mathrm{O}^{\mathrm{O}}=$ Homophilie dyadique

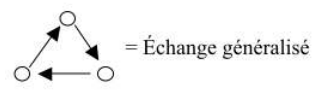

Pour ce qui est du statut culinaire, l'homophilie n’est que partiellement vérifiée (sous-structure dyadique), ce qui est dû, selon nous, à la position intermédiaire de ce capital dans la structure du champ, dont la possession est nécessaire mais non suffisante dans la concurrence pour l'accession à la reconnaissance gastronomique. Ces restaurateurs qui ne disposent que d'un capital culinaire peuvent être considérés comme des aspirants à la reconnaissance gastronomique. Cela se ressent dans la manière dont ils mènent leur travail relationnel. S'ils recherchent le contact avec leurs semblables, ils n'en restent pas moins conscients de la concurrence symbolique à laquelle ils se livrent, c'est une interprétation possible de l'absence de cycle entre eux. À propos, enfin, des restaurateurs disposant du seul statut économique,

24 Ou «échange généralisé» selon l'expression de Levi-Strauss, qui constitue une forme de réciprocité indirecte. 
on constate un effet significatif et négatif des sous-structures dyadique et triadique. Ces restaurateurs, dont le seul atout repose sur la taille du restaurant et les parts de marché, sont d'autant plus sensibles à la concurrence économique entre pairs, et ont donc tendance à éviter les interactions homophiles, voire les interactions tout court (car on l'a vu dans le tableau 3 , ils en ont très peu).

\section{CONSTRUCTION ET HIÉRARCHIE DES NICHES SOCIALES}

Le troisième type d'analyse que nous développons concerne la construction de niches sociales. Lors de la passation du questionnaire, nous avons constaté que l'existence, entre deux restaurateurs, d'échanges d'informations intéressantes est significative d'un lien qui dépasse le cadre de la simple relation d'interconnaissance, et qui s'accompagne généralement d'un lien d'amitié. C’est pourquoi nous nous focalisons plus spécifiquement sur ce réseau social qui compte 73 I liens entre 283 restaurateurs (soit une densité de $\mathrm{I} \%{ }^{25}$ ) et dont $30 \%$ des liens sont réciproques ${ }^{26}$. Lorsqu'on dessine ce réseau, où chaque restaurateur est représenté par un point et où chaque transfert d'informations est représenté par une flèche, on obtient un graphe incompréhensible (voir la figure 2). Un outil d'analyse de réseau, appelé blockmodel, permet de simplifier cette figure en faisant ressortir les sous-groupes et les liens entre ces sous-groupes. Nous avons réalisé le blockmodel du réseau d'informations (voir la figure 3) ${ }^{27}$. Sa particularité est de compter cinq blocks (où les points ne représentent plus des restaurateurs mais des sous-groupes de restaurateurs), et que ces cinq blocks ne sont pas reliés entre $\operatorname{eux}^{28}$, ce qui implique que quand des informations intéressantes circulent, elles ont tendance à le faire au sein de sous-groupes relativement circonscrits. Ces blocks ne sont pas forcément tous des niches sociales. Pour repérer ces niches, nous posons deux conditions : d’une

25 La densité est le nombre de relations observées divisé par le nombre de relations possibles.

26 C'est-à-dire que lorsqu'un restaurateur affirme échanger des informations avec un autre restaurateur, dans 30\% des cas, la réciproque est vraie.

27 Pour de plus amples explications concernant la mise en œuvre de notre blockmodel, voir Éloire (2009).

28 Le tableau des densités du blockmodel fait apparaître des densités inter-blocks très faibles (la plus élevée : 0,245), signe que les blocks entre eux communiquent peu, tandis que les densités intra-blocks sont, elles, significativement plus élevées (voir le tableau 5). 
part, ils doivent avoir une densité élevée (le nombre de relations observées entre les membres d'un sous-groupe est-il important?), et d'autre part, l'échange généralisé doit être significativement et positivement présent (le paramètre «cycle», comme expression d'une réciprocité indirecte entre les membres du sous-groupe, est-t-il significatif et positif?). Sur les cinq blocks, deux d'entre eux vérifient ces conditions et peuvent donc être qualifiés de niches sociales (voir le tableau 5).

Figures 2 et 3 : le réseau d'informations intéressantes et son blockmodel
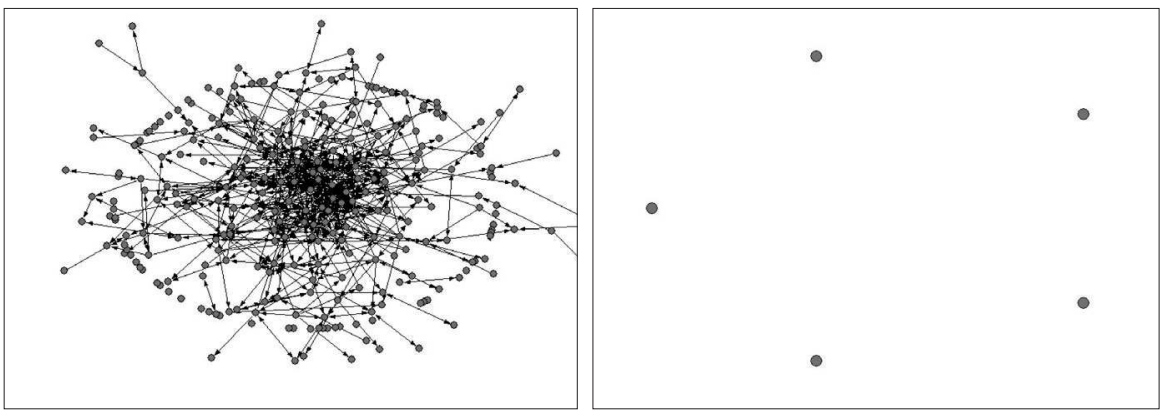

Source: Éloire, 2008. Logiciels Pajek (dessin) et Ucinet (blockmodel).

Tableau 5 : le repérage des niches sociales parmi les cinq blocks

\begin{tabular}{|c|c|c|c|c|}
\hline BLOCKS & $\begin{array}{c}\text { NB DE } \\
\text { RESTAURATEURS }\end{array}$ & $\begin{array}{c}\text { DENSITÉ } \\
\text { INTRA-BLOCKS }\end{array}$ & $\begin{array}{c}\text { CYCLE } \\
(*=\text { SIGNIFICATIF })\end{array}$ & NICHE SOCIALE \\
\hline 1 & 40 & 1,956 & $+0.855(0.033)^{*}$ & Oui \\
\hline 2 & 40 & 1,808 & $+0.284(0.019)^{*}$ & Oui \\
\hline 3 & 60 & 1,017 & $+0.173(0.058)$ & Non \\
\hline 4 & 70 & 1,944 & $-0.354(0.023)^{*}$ & Non \\
\hline 5 & 63 & 0 & - & Non \\
\hline
\end{tabular}

Source: Éloire, 2008.

Pour faire le lien entre cette recherche des niches sociales et celle du champ gastronomique, nous projetons l'appartenance à l'un des cinq blocks (devenu attribut individuel) dans l'ACM. Une hiérarchie sociale des blocks s'établit du block 5, situé en bas du plan et qui rassemble en fait les restaurateurs isolés au sein du réseau d'informations (ceux qui ne pratiquent jamais l'échange d'informations) aux blocks I et 2, situés en haut à droite du plan et que nous avons repérés comme 
étant des niches sociales (voir la figure 4). Les restaurateurs qui construisent des niches sociales sont donc aussi ceux qui sont les mieux dotés en capital culinaire et capital gastronomique, ce qui est selon nous une autre manifestation de la manière dont la gastronomie se construit socialement.

Ainsi, bien qu'il soit uniquement fondé sur des données d'interactions, le blockmodel révèle donc sa signification dès lors qu'on rapproche son résultat de celui du champ. D'une part, les échanges d'informations ne s'effectuent pas au hasard entre les restaurateurs. D’autre part, la niche sociale $\mathrm{n}^{\circ}$ I étant située au-dessus de la niche sociale $\mathrm{n}^{\circ}$ 2, il semble s'établir une hiérarchie entre les deux niches, fondée sur le volume de capital culinaire et gastronomique des membres qui les composent : la niche $\mathrm{n}^{\circ}$ I rassemble la plupart des oligarques multi-statutaires, et la niche $\mathrm{n}^{\circ}$ 2, les «aspirants». En réalité, derrière la solidarité limitée qu'exprime la niche sociale, se dévoile le caractère stratégique de ce phénomène : les échanges de ressources sont vecteurs de coopération, mais sont aussi des instruments de concurrence de statut entre groupes plus ou moins rivaux.

Figure 4: Hiérarchie des niches sociales du réseau d'informations au sein du champ de la restauration lilloise

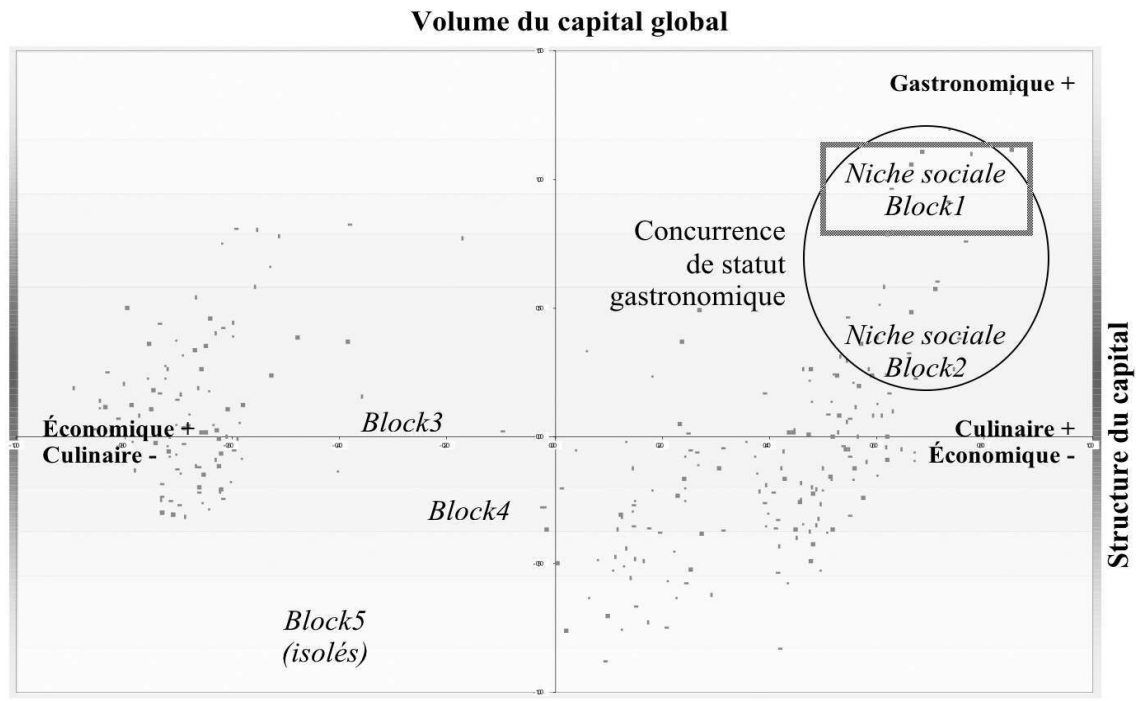

Source: Éloire, 2008. 
Pour comprendre la logique sous-jacente de la construction de ces niches, on peut étudier les liens d'affiliation aux associations «honorifiques», qui constituent les révélateurs de cette concurrence de statut. Bien qu'ils restent peu répandus (seuls $17 \%$ des restaurateurs de notre population sont affiliés à une association honorifique), ces liens d'affiliation sont porteurs de significations sociales et professionnelles fortes puisque ces associations constituent un vecteur essentiel de la construction de la profession et, à l'intérieur même de celle-ci, de la «qualité » des produits et de leurs producteurs. L'association qui est certainement la plus centrale de cette étude s'appelle les Tables Gourmandes. Elle a été créée en 1999 à l'initiative de Chefs installés sur la métropole lilloise. Son action est exclusivement locale : elle vise principalement à promouvoir la gastronomie locale. Elle compte une trentaine de membres, tous Chefs, à la tête d'établissements proposant une cuisine de style gastronomique ou semigastronomique. Les réunions et actions de cette association sont l'occasion pour ces Chefs de communiquer, ce qui facilite entre eux les échanges de ressources sociales tels que nous les avons étudiés. Pourtant, contrairement à ce qu'on aurait pu imaginer, les membres de cette association ne sont pas regroupés dans une même niche sociale, mais se répartissent, de manière à peu près égale, entre les deux niches sociales (I5 restaurateurs dans la niche $\mathrm{n}^{\circ}$ I, et I3 dans la $\mathrm{n}^{\circ} 2$, voir le tableau 6). Ce qui montre que la coappartenance formelle à une association ne garantit pas l'échange informel de ressources sociales inconditionnel entre ses membres. Le Club des Tables Gourmandes est en réalité traversé par la concurrence de statut. Comme le montre encore le tableau 6, ce qui explique, en fait, l'appartenance à l'une ou à l'autre des deux niches sociales, c'est l'affiliation à une autre association, non plus locale, mais nationale. Sur ce marché, nous en avons dénombré cinq: les Disciples d'Escoffier, Prosper Montagné, Générations.C, les Jeunes Restaurateurs d’Europe, les Maîtres cuisiniers de France. Tous les restaurateurs (à part un) qui, en plus des Tables Gourmandes, appartiennent à une association honorifique nationale se rassemblent informellement dans la niche sociale la plus prestigieuse (la $\left.\mathrm{n}^{\circ} \mathrm{I}\right)$. 
Les affiliations à ces associations honorifiques nationales fonctionnent sur la base de la volonté individuelle, mais l'affiliation n'est pas libre, c'est-à-dire pas uniquement conditionnée au règlement d'une cotisation; elle est soumise à approbation des autres membres de l'association: des règles de cooptation des nouveaux membres sont institutionnalisées, qui permettent une sélectivité, garantie de «qualité » sociale et professionnelle des restaurateurs cooptés. La démarche est toujours à peu près la même : d'une part, il faut justifier d'une «solide expérience professionnelle», justification qui peut même donner lieu au remplissage d'«un questionnaire soumis à une commission d'enquête »; d’autre part, il faut être parrainé par deux membres de l'association; cette condition traduit à elle seule l'importance des réseaux sociaux entre professionnels sur le marché gastronomique. Ces associations véhiculent des conceptions différentes de la gastronomie se proposant, par exemple, tantôt, dans une veine traditionnaliste, de «préserver et transmettre l'héritage immense de l’art culinaire » (Maîtres Cuisiniers de France), tantôt, dans une veine modernisatrice, de «tracer les nouvelles voies de la cuisine française» (Générations. C). Mais ces divergences ne doivent pas masquer la complicité objective d’adhérents à des associations concurrentes qui, en réalité, comme le montre l’analyse de réseau, s'échangent volontiers informellement des ressources sociales. Le statut que confère l'affiliation honorifique joue ici comme un signal de reconnaissance et prime objectivement sur les conflits idéologiques. On peut penser qu'en construisant ensemble cette niche sociale prestigieuse (niche $\mathrm{n}^{\circ} \mathrm{I}$ ), les oligarques multi-statutaires cherchent à entretenir et à reproduire un statut qui leur donne l'avantage de définir eux-mêmes les contours de ce qu'est la «gastronomie », excluant de leurs échanges des restaurateurs, certes moins prestigieux (niche $\mathrm{n}^{\circ} 2$ ), mais potentiels aspirants à la reconnaissance gastronomique. 
Tableau 6: Concurrence de statut au sein des niches sociales à travers le prisme des liens d'affiliations honorifiques

\begin{tabular}{|c|c|c|c|}
\hline & $\begin{array}{c}\text { NICHE SOCIALE N } \\
(\mathrm{N}=40)\end{array}$ & $\begin{array}{c}\text { NICHE SOCIALE N } \\
(\mathrm{N}=40)\end{array}$ & $\begin{array}{c}\text { AUTRES BLOCKS } \\
=200\end{array}$ \\
\hline TABLES GOURMANDES & 15 & 13 & 1 \\
\hline PROSPER MONTAGNÉ & 5 & 1 & 0 \\
\hline DISCIPLES D'ESCOFFIER & 9 & 1 & 0 \\
\hline $\begin{array}{c}\text { GÉNÉRATIONS. C } \\
\text { JEUNES RESTAURATEURS } \\
\text { D'EUROPE }\end{array}$ & 3 & 0 & 0 \\
\hline MAîTRES CUISINIERS DE FRANCE & 1 & 0 & 0 \\
\hline
\end{tabular}

Source: Éloire, 2008.

\section{CONCLUSION}

Les trois types d'analyses de réseau cherchent à montrer à l'œuvre le capital social des restaurateurs. Ce capital social agit inséparablement au niveau individuel et au niveau collectif. Alors que la centralité est fondamentalement un indicateur de prestige individuel, l'homophilie et les niches sociales sont fondamentalement des constructions collectives. Comme le montrent nos analyses, les niches sociales sont construites par ceux qui sont aussi les plus centraux et cette construction s'effectue entre des restaurateurs qui ont des intérêts communs, comme celui de faire exister le marché de la gastronomie lilloise. Alors que le comportement des restaurateurs de statut uniquement économique semble plus proche de celui de l'homo œeconomicus décrit par la théorie néoclassique, il ne constitue qu’un cas particulier du possible des comportements de restaurateur. La construction sociale de la qualité est marquée par l'existence des établissements scolaires qui dispensent une formation en hôtellerie-restauration, ainsi que par l'effet que produisent les «dispositifs de jugement» (Karpik, 2007) que sont les guides gastronomiques. Mais l'étude de 
ce milieu professionnel, à travers les échanges de ressources, montre comment s'effectue la reproduction de la structure hiérarchique du champ gastronomique.

Les trois formes de capital, économique, culinaire et gastronomique, impliquent des rapports sociaux de concurrence et de domination que l'analyse en termes de champ objective. Mais c'est dans la structure des interactions, c'est-à-dire dans le travail relationnel qui est effectué par les restaurateurs, que ces rapports opèrent. Et c'est l'analyse de réseau qui permet de révéler le lien entre les deux types de relations sociales, autrement dit entre les structures objectives et les interactions intersubjectives. Le principal résultat est, selon nous, d’avoir montré le rapport entre la pratique de la gastronomie et les échanges de ressources sociales entre pairs. Dans ce contexte, les réseaux d'interconnaissance et d'inter-reconnaissance sont d'autant plus importants dans l'activité économique que le motif purement économique, à savoir la réalisation du profit, ne peut pas toujours être une fin en soi. Ainsi, on peut affirmer quau sein du marché de la restauration, l'économie «ordinaire» côtoie une «économie à l'envers», selon l'expression de Bourdieu décrivant le fonctionnement du «marché des biens symboliques» (1992b, p. 20I et suiv.). «C'est en produisant la rareté du producteur que le champ de production symbolique produit la rareté du produit (...) [ainsi], toute théorie économique de la production de biens symboliques est fausse qui ne prend en compte que les coûts de fabrication des objets considérés dans leur matérialité» (Bourdieu, Delsaut, 1975, p. 21).

Dans la prise en compte des «autres coûts», qui sont liés à l'ancienneté, à la capacité de durer et de «faire date», la description du processus d'accumulation du capital social joue un rôle important. Certes, le capital social a un rôle instrumental et stratégique : il est «utile» et sert des «intérêts». Mais, ces intérêts ne sont pas qu'économiques et matériels, ils sont aussi sociaux et symboliques, aspects pour l'analyse desquels la sociologie économique est bien outillée. Notre volonté, dans ce travail, était, tout en dialoguant avec l'économie standard, de décentrer notre regard des aspects strictement marchands de l'activité économique vers les aspects non marchands. Cette étude constitue, ainsi, une illustration de la «métaphore de l'encastrement» posée par Karl Polanyi (1944, 1957) pour nous rappeler que toute société a une économie, et toute économie ne peut se développer que dans une société. 


\section{BIBLIOGRAPHIE}

BOURDIEU P., DELSAUT Y., 1975, «Le couturier et sa griffe: contribution à une théorie de la magie», Actes de la Recherche en Sciences Sociales, n I, p. 7-36.

-, 1979, La Distinction, Paris, Éditions de Minuit.

-, 1980, «Le capital social, notes provisoires», Actes de la Recherche en Sciences Sociales, n०31, p.2.

-, 2002 [1984], «Quelques propriétés des champs», in Questions de sociologie, Paris, Éditions de Minuit, p. I13-120.

-, 1992, Réponses: pour une anthropologie réflexive, Paris, Seuil.

-, 1992b, Les Règles de l'art. Genèse et structure du champ littéraire, Paris, Seuil.

-, 1994, Raisons pratiques. Sur la théorie de l'action, Paris, Seuil.

-, 1999, «Une révolution conservatrice dans l'édition», Actes de la Recherche en Sciences Sociales, n 126-127, p. 3-28.

-, 2000, Les Structures sociales de l'économie, Paris, Seuil.

-, 2007, «La noblesse: capital social et capital symbolique», in D. Lancien, M. Saint-Martin, Anciennes et nouvelles aristocraties de 1880 à nos jours, Paris, Éditions de la Maison des Sciences de l'Homme.

BOYER R., FREYSSENET M., 2000, Les Modèles productifs, Paris, La Découverte, «Repères».

-, 2003, «L'anthropologie économique de Pierre Bourdieu», Actes de la Recherche en Sciences Sociales, n I50, p. 65.

-, 2009, «Marché, État et capitalismes», in Steiner et Vatin, Traité de sociologie économique, Paris, PUF, p. 53-87.

BURT R., 1995, «Le capital social, les trous structuraux et l'entrepreneur», Revue Française de Sociologie, 36, p. 599-628.

-, 2005, Brokerage and closure, an introduction to social capital, Oxford, Oxford University Press.

-, 2008, «Gossip and Reputation », in Management et réseaux sociaux, ressource ou outil de gestion, London, HERMES Science Publishing Ldt.

CASTORIADIS C., 1999, «La "rationalité" du capitalisme», in Les Carrefours du labyrinthe, 6, Figures du pensable, Paris, Seuil, «Points Essais», p. 79-।I2. 
COLEMAN J., 1988 «Social capital in the creation of human capital», American Journal of Sociology, n॰94, p. 95-120.

CONVERT B., HEILBRON J., 2004, «Genèse de la "nouvelle sociologie économique" aux États-Unis», in J. Heilbron, R. Lenoir, G. Sapiro, Pour une histoire des sciences sociales. Hommage à Pierre Bourdieu, Fayard, p. 223-24l.

DALLERY Th., ÉLOIRE F., MELMIÈS J., 2009, «La fixation des prix en situation d'incertitude et de concurrence: Keynes et White à la même table», Revue Française de Socio-économie, «Sciences sociales et pratiques économiques », $2^{\mathrm{e}}$ semestre, n4, Paris, La Découverte, p. 177-198.

-, 2010, «L'incertitude au cœur des marchés concrets. Confrontation entre théorie post-keynésienne et sociologie économique structurale», Innovations. Cahiers d'Économie de l'Innovation, n³1, p. 131-156.

DALLERY T., VAN TREECK T., 2008, «Conflicting Claims and Equilibrium Macroeconomic Adjustment Processes in a Stock-Flow Consistent Macromodel », IMK Working Paper, n॰9, [s.p.].

DE NOOY W. 2003, «Fields and networks: correspondence analysis and social network analysis in the framework of field theory », Pœtics, n³1, p. 305-327.

DUVAL J., 2000, «Concessions et conversions à l'économie. Le journalisme économique en France depuis les années $1980 \gg$, Actes de la recherche en sciences sociales, $n^{\circ}$ |31-132, p.56-75.

ÉLOIRE F., 2009, «Le rôle des réseaux sociaux dans l'activité économique: le cas des restaurateurs lillois », REDES, revista hispana para el anàlisis des redes sociales, vol. 16/8, p.203-227 - http://revista-redes.rediris.es/html-voll6/voll6_8f.htm.

FRANÇOIS P., 2009, Sociologie des marchés, Paris, Armand Colin.

GISLAIN J. J., STEINER Ph., 1995, La Sociologie économique, 1890-1920, Paris, PUF.

GLASER B. G., STRAUSS, A.L., 1967, The Discovery of Grounded Theory: strategies for qualitative research, Piscataway (N.J.), Aldine Transaction.

GODECHOT O., MARIOT N., 2004, «Les deux formes du capital social. Structure relationnelle des jurys de thèse et recrutement en science politique », Revue Française de Sociologie, vol. 45, n², p. 243-282. 
GRANOVETTER M., 1973, "The strength of weak ties", American Journal of Sociology, 78/6, p. 1360-1380. Traduction en français in Le Marché autrement, 2000, Paris, Desclee de Brouwer.

GUERRIEN B., 2002, Dictionnaire d'analyse économique, microéconomie, macroéconomie, théorie des jeux, etc., Paris, La Découverte, «Repères», p. 21 .

KARPIK L., 2007, L'Économie des singularités, Paris, Gallimard, «NRF».

LAZEGA E., 2007 [1998], Réseaux sociaux et structures relationnelles, Paris, PUF, «Que sais-je».

-, 1999, «Le phénomène collégial: une théorie structurale de l'action collective entre pairs», Revue Française de Sociologie, II / 4, p. 639-670.

-, 200I, The Collegial Phenomenon, the social mechanisms of cooperation among peers in a corporate law partnership, Oxford, Oxford University Press.

-, MOUNIER L., 2002, "Interdependent entrepreneurs and the social discipline of their cooperation: The research program of structural economic sociology for a society of organizations", in Favereau, Lazega (eds), Conventions and Structures in Economic Organization: Markets, Networks, and Hierarchies, Cheltenham, Edward Elgar Publishing, p. 147-199.

-, 2003, «Rationalité, discipline sociale et structure», Revue Française de Sociologie, 44-2, p. 305-329.

-, 2005, «Capital social, processus sociaux et capacités d'action collective», in A. Bevort, M. Lallement, Le Capital social - Performance, équité et réciprocité, Paris, La Découverte.

-, 2009, «Théorie de la coopération entre concurrents: organisations, marchés et réseauX», in Steiner et Vatin, Traité de sociologie économique, Paris, PUF, p. 533-57I.

-, LEBEAUX M.-O., 1999, «Capital social et contrainte latérale», Revue Française de Sociologie, 36, p.759-777.

-, JOURDA M.T., MOUNIER L., STOFER R., 2007, «Des poissons et des mares: l'analyse de réseau multi-niveaux», Revue Française de Sociologie, vol. 48, n I.

LEBARON F., 1997, «La dénégation du pouvoir. Le champ des économistes français au milieu des années 1990 », Actes de la Recherche en Sciences sociales, n० 119, p. 3-26. 
LEVESQUE B., BOURQUE G., FORGUES E., 200I, La Nouvelle sociologie économique: originalité et diversité des approches, Paris, Desclée de Brouwer.

LIN N., 1995, «Les ressources sociales: une théorie du capital social», Revue Française de Sociologie, 36, p. 685-704.

POLANYI K., 2005 [1944], La Grande Transformation: aux origines politiques et économiques de notre temps, Paris, Gallimard.

-, 1957 [1975], «L'économie en tant que procès institutionnalisé », in K. Polanyi, C. Arensberg, Les Systèmes économiques dans l'histoire et dans la théorie, Paris, Larousse.

PONTHIEUXS., 2006, Le Capital social, La Découverte, «Repères ».

PUTNAM R., 1993, "The prosperous community. Social capital and public life", The American Prospect, vol. 4, n¹3, p. 35-42.

SAPIRO G., 1996, «La raison littéraire, le champ littéraire français sous l'Occupation (1940-1944) », Actes de la Recherche en Sciences Sociales, n | | | - | I2, p. 3-35.

STEINER Ph., 1999, La Sociologie économique, La Découverte, «Repères».

-, 2005, «Le marché selon la sociologie économique», Revue Européenne des Sciences Sociales, Tome XLIII, n 132, p. 31-64.

SWEDBERG R., 1994, Une histoire de la sociologie économique, Paris, Desclée de Brouwer.

VERGER A., 1991, «Le champ des avant-gardes», Actes de la Recherche en Sciences Sociales, $n \circ 88$, p. $2-40$.

WASSERMAN S., FAUST, K., 1994, Social network analysis: methods and applications, Cambridge, Cambridge University Press.

WHITE H., 1981, "Where do markets come from?", American Journal of Sociology, $87 / 3$, p. $517-587$.

-, 2002, Markets from networks. Socioeconomic models of production, Princeton, Princeton University Press. 
